\title{
Examination of an Audiologist's Response to Patient's Expression of Symptoms: A Pilot Study
}

\author{
Ashley L. Dockens ${ }^{1}$, Monica L. Bellon-Harn ${ }^{1}$, Erin S. Burns ${ }^{1}$, \\ Vinaya Manchaiah ${ }^{1,2,3}$, and Orlando Hinojosa ${ }^{1}$ \\ ${ }^{1}$ Department of Speech and Hearing Sciences, Lamar University, Beaumont, TX, USA \\ ${ }^{2}$ The Swedish Institute for Disability Research, Department of Behavioural Sciences and Learning, Linköping University, \\ Linköping, Sweden \\ ${ }^{3}$ Audiology India, Mysore, Karnataka, India
}

Received November 14, 2016

Revised January 20, 2017

Accepted February 27, 2017

Address for correspondence

Monica L. Bellon-Harn, PhD,

CCC-SLP

Department of Speech and

Hearing Sciences, Lamar University,

Beaumont, TX 77710, USA

Tel $+1-409-880-7655$

Fax $+1-409-880-2265$

E-mail monica.harn@lamar.edu
This pilot study explores audiologist-patient interactions during initial evaluations or consultations. In particular, an audiologist's response to patient symptoms is examined. Conversations between audiologist and patients were recorded using a digital recorder, which were transcribed, and analyzed using the Codes for Human Analysis of Transcripts and Child Language Analysis computer programs. Mean length of turn and frequency of utterances related to explicit discussion or description of symptoms or the patient's interpretation of symptoms was determined. Study sample: six audiologist-patient interactions were recorded and transcribed. A single audiologist was used for this pilot investigation. Results suggest that during the initial audiological consultations related to hearing difficulties the audiologist produced more utterances related to explicit description of the symptoms, whereas when during the sessions about complex disorder and hearing aid consultation the audiologist produced more utterances related to the patient's interpretation of the symptoms. Also, a more equitable distribution of words and utterances per turn are observed during the initial consultation about hearing difficulties when compared to complex disorders and hearing aid consultation sessions where the audiologist was dominant within the interaction. This preliminary study reveals unique insights to audiologist's communication behavior during audiology consultation session. Efforts are needed to educate and promote appropriate communication between audiologists' and patients, which could result in increased patient satisfaction.

J Audiol Otol 2017;21(2):115-119

KEY WORDS: Patient-centeredness · Health communication · Psychosocial Interactional analysis · Rehabilitation.

\section{Introduction}

Communicative interactions in healthcare settings should be satisfying to the patient in order to lead to improved care and outcomes for the patient [1]. Practitioners can employ communicative strategies to promote effective and reciprocal interactions that are meaningful based on the patient's frame of reference [2]. One area in which effective communication may lead to improved health outcomes is when it is used for

This is an Open Access article distributed under the terms of the Creative Commons Attribution Non-Commercial License (http://creativecommons.org/licenses/by-nc/4.0/) which permits unrestricted non-commercial use, distribution, and reproduction in any medium, provided the original work is properly cited. correct diagnosis and treatment decisions [3]. Within the profession of audiology, this is a critical issue since untreated hearing loss impacts individuals with hearing loss and their families, communities, and society, psychologically, socially, cognitively, physically, and financially [4]. To have correct diagnosis and management, audiologists must match patient experiences with their symptoms appropriately, which requires effective communication. Effective communication is a necessary skill that enables the healthcare provider to best understand the illness from a patient point of view [5]. While Brown, et al. [5] suggest this communication will be straightforward; the provider must be cued into feelings, ideas, effect on function, or expectation. 
Listen to the patient and understand their concerns and symptoms may be key components of the interaction. To accomplish this type of communication, the audiologist must open the floor to the patient to allow for equitable contribution and mutual sharing of ideas. Mutual influence in conversation facilitates shared decision-making and increases conversational reciprocity [6]. Additionally, audiologists should use partnership-building behaviors more frequently such as open-ended questions [7]. For example, audiologists must move beyond asking their patient, "Do you have any questions?", to allowing the patient to actively communicate and give feedback regarding their level of understanding.

Open-ended questions evoke longer responses that allow the patient to communicate with his or her words with longer utterances. Additionally, patients may benefit when audiologists actively listen and respond empathetically to feelings. However, a series of recent reports examining audiologist-patient conversations indicate that audiologists infrequently engage in such communication [8-10]. Consequently, there is little relationship building. For this reason, education and practice efforts are needed to implement effective communication within audiology appointments.

In an effort to explicate moment-by-moment interactions, this pilot study explores audiologist-patient interactions during initial evaluations or consultations. In particular, an audiologist's response to patient symptoms was examined. The following questions were asked:

1) Does the frequency of utterances that explicitly discuss symptoms or the experience of the impairment related to the symptoms differ relative to appointment type?

2) Does the distribution of conversational load between the audiologist and patient during interactions differ relative to appointment type?

\section{Subjects and Methods}

In order to address the research questions, qualitative and quantitative analyses of interactions between a single audiologist and six different patients were conducted. A single audiologist was used for this pilot investigation to limit concerns for variation across clinical behavior. While this diminishes generalization, the study will determine the utility of the research tools and procedures used for evaluating clinical behavior across a greater number of audiologists. The audiologist used in this study has an exceptionally low hearing aid return-for-credit rate of $2 \%$ for the tracking time period of 2009 to 2016. For this reason, the audiologist is considered successful in obtaining patient compliance since the percentage is significantly lower than national average (i.e., 20.1\%)
[11]. An audiologist successful in this area was desirable for this research to limit variability potentially seen with less successful audiologists.

Patient inclusion was based on physician and self-referral to the university speech and hearing clinic at which the target audiologist is employed. Ethical approval was obtained from the Research Ethics Committee, Lamar University (IRB Approval \#7341637). Clinical appointments were selected based on convenience and included initial evaluations or hearing aid consultations. Long-term patients were excluded from the study (i.e., those who have consulted audiologists several times within the last three months).

\section{Procedure}

Six audiologist-patient interactions were recorded, transcribed, and included in the analysis. Session 1 included an adult seeking an initial evaluation for symptoms related to hearing difficulties and tinnitus. Sessions 2, 3, and 4 included adults who were seeking an initial evaluation related to hearing difficulties alone. Session 5 included an adult with a history of auditory processing disorder seeking an initial evaluation for hearing loss. Session 6 included an adult seeking an initial consultation for hearing aid use. Samples were collected using an Olympus digital voice recorder (DM-620; Olympus, Tokyo, Japan) and Olympus stereo microphone (ME-51S; Olympus). The audiologist obtained consent from each patient, and attached the recorder to her lab coat. The session was recorded, transcribed, and analyzed using computer programs within the TalkBank system. These included Codes for Human Analysis of Transcripts and Child Language Analysis (CLAN) [12]. These programs provide tools to code and analyze conversational interactions within any dyad (e.g., clientpractitioner, family-practitioner).

Following transcription, content analyses of audiologistpatient exchanges were conducted. Utterances were coded for whether the audiologist explicitly discussed or described symptoms or discussed the patient's interpretation of the impairment or not. Within the exchanges, distribution of conversational load was analyzed using mean length of turn (MLT) measures from the CLAN computer program. Conversational load refers to the amount of talk each participant uses within each turn. Analysis of MLT affords a quantitative analysis of the degree of balance within an interaction by measuring the total number of utterances and words per conversational turn.

Next, the utterances within the exchanges were segmented. Utterances consisted of a complete thought. Each utterance produced by the target audiologist was coded as either discussing and/or describing the symptom or the patient's inter- 
pretation of the symptom. The Measure of Patient-Centered Communication (MPCC) was used as a guide to define utterances related to the either category [5]. The MPCC is a tool that reflects the exploration of health and disease, how the concepts of health and disease integrate with the whole person, and finding common ground with the patient. The MPCC uses six distinct components to measure the patientcenteredness of utterances made by providers and patients [5]. The present research is concerned with the expression of symptoms related to hearing loss. As such, the coding categories within the first component of the MPCC (i.e., exploration of health and disease) were used to identify the audiologist's utterances regarding explicit discussion of symptoms or the patient's interpretation of the symptoms. As noted by Epstein, et al. [13], there should be clarity of the patient-centered construct being measured. For the purposes of this study, the MPCC provided a guide to explicitly define the variable of interest.

An utterance was coded as explicitly discussing or describing the symptom if the utterance consisted of: 1) a direct statement or questions related to the symptom (e.g., "When was your last ear infection?"), 2) an out of context statement but related to patient concerns (e.g., "Some people only get dizzy when they turn left in bed."), or 3) a restatement of the problem that has already been mentioned (e.g., That makes sense so you, your ears start ringing, you get headaches). An utterance was coded as a discussion of the patient's interpretation of the symptom if the utterance included a question or statement related to feeling, ideas, or effect on function (e.g., "Let's analyze the problem. What's making it hard to hear?").

A total of 327 utterances were coded. Frequency of utterances related to explicit discussion and/or description of symptoms or the patient's interpretation of symptoms was determined using the CLAN computer programs [12]. Percent occurrences of utterances related to description or the patient's interpretation of symptoms were calculated.

\section{Reliability}

Two trained researchers completed all transcription. First, each researcher transcribed and coded the samples individually using the conversational analysis font within the CLAN computer program. Then, samples from both were compared. If agreement could not be reached on all utterances, the second author listened, transcribed, and coded the utterances in question to make the final decision. They segmented the transcript into target utterances. To establish utterance segmentation reliability, the first author evaluated $20 \%$ of the full corpus of 198 utterances. Utterances were compared using verbatim agreement in that every word within each utter- ance had to be the same. Agreement was calculated using the following formula: $100 \times$ agreements/(agreements + disagreements). Utterance segmentation reliability was 95\%. The trained researchers coded the samples. The procedure presented previously was used to ensure consistency and reliability. Utterances were compared using verbatim agreement in that every code had to be the same. Agreement was calculated using point-by-point comparison and the following formula: $100 \times$ agreements/(agreements + disagreements). Agreement for coding was $92 \%$.

\section{Results}

Results of the sample analyses are presented below according to utterances characterized as explicit discussion of symptom or discussion of the patient's interpretation of the symptom and mean length of audiologist and patient turns. During sessions in which an adult sought an initial evaluation related to hearing difficulties (i.e., sessions 1 to 4 ) the audiologist produced more utterances related to explicit description of the symptoms (Fig. 1). During the session in which the presenting concern of hearing difficulties including a history of auditory processing disorder and the sessions in which the adult was seeking initial hearing aid consultation (i.e., sessions 5 and 6), the audiologist produced more utterances related to the patient's interpretation of the symptoms.

During sessions in which an adult sought an initial evaluation related to hearing difficulties (i.e., sessions 1 to 4 ) a more equitable distribution of words and utterances per turn are observed than during other sessions (Fig. 2). During session 5, in which the presenting concern of hearing difficulties with history of auditory processing disorder, and session 6 , in which the adult was seeking initial hearing aid consultation,

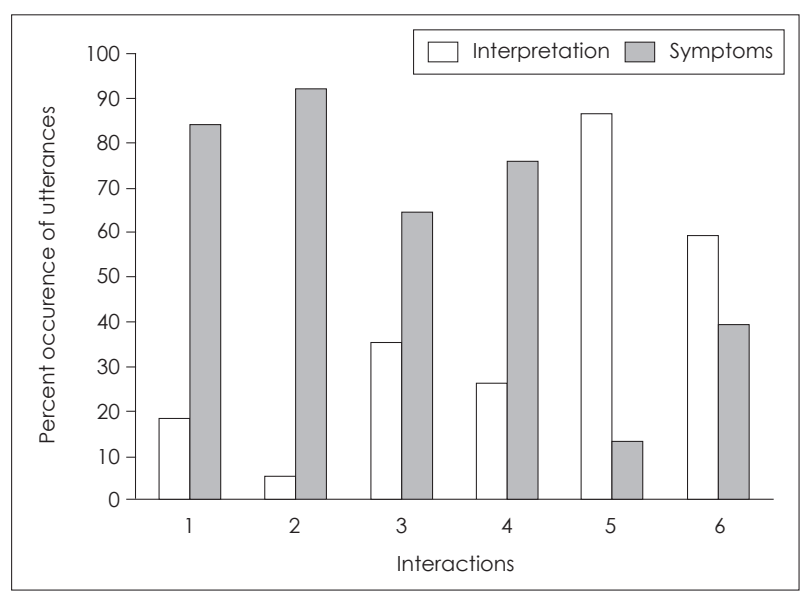

Fig. 1. Percent of audiologist's utterances related to explicit discussion of symptom or discussion of the patient's interpretation of the symptom. 


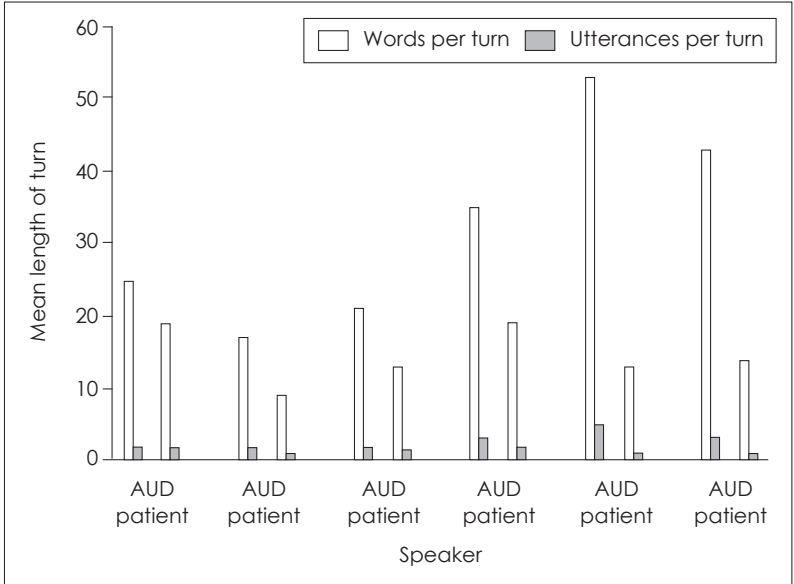

Fig. 2. Mean length of turn measures for each audiologist (AUD) and patient dyad.

the audiologist was dominant within the interaction.

\section{Discussion}

The current study explores audiologist-patient interactions during initial evaluations or consultations. It is evident from the study results that the audiologist communication behavior differs between initial consultation for routine hearing evaluation session when compared to other types of sessions (e.g., complex hearing disorders or hearing aid consultation). Frequency of utterances that explicitly discuss symptoms or the experience of the impairment related to the symptoms and distribution of conversational load differed relative to appointment type. The tools and procedures utilized in this study appropriately captured the audiologist response to patient symptoms.

Previous studies indicate that there is a tendency for audiologists to control the agenda during initial consultations [9]. The audiologist in this study was more dominant across all initial conversations. However, more words and utterances per turn were noted during the interaction involving complex cases and hearing aid consultation. This finding suggests that greater diagnostic and management complexity may warrant increased conversational control on the part of the audiologist.

Additionally, it is reported that audiologists tend to use closed ended questions, which does not help in building relationships with patients $[9,10]$. In this study, the audiologist controlled the majority of interactions. Had more open ended questions been used, it is expected that the patient would be a more active participant in the interaction. As noted, this type of communication gap can also result in poor adherence and need for greater number of interactions between audiologists and patients during management of hearing loss [8]. As noted recently, the majority of audiologists' education and counseling utterances were practitioner-centered, related to treatment with hearing aids, but only $56 \%$ of patients decided to obtain hearing aids [10]. These studies clearly demonstrate the need for audiologists to adopt better balance and more appropriate communication during audiological consultation.

Although the audiologist in this study was more dominant during the interaction involving complex cases and hearing aid consultation, more attention to the experience of the patient occurred during these interactions than during an initial evaluation related to hearing difficulties. As noted by Brown, et al. [5], effective communication includes understanding the patient's point of view. It may be that initial evaluations require greater description of the symptoms, whereas initial consultations regarding complex issues or with patients who have experienced hearing aids instead require more consideration of the patient's experiences. In both, the audiologist must be cued into feelings, ideas, effect on function, and/or expectations. However, a more explicit approach could be beneficial during initial evaluations.

In an ideal clinical scenario, balance in communication is needed between various aspects such as symptoms description, interpretation of symptoms, addressing psychological concerns and so on. Based on these analyses, the balance in conversations and type of utterance may be dependent on the need of the patient and goal of the visit. Patients may want audiologists to take the lead in: 1) explaining the severity of the condition (e.g., type and degree of hearing loss); providing on communication strategies; 2) discussing rehabilitation (e.g., amplification) options based on symptoms; and 3) possible outcome of the rehabilitation. In addition, patients may also expect audiologists to address their concerns. However, to appropriately take lead, an audiologist must first have a complete understanding of symptoms and related patient experiences. Understanding how to negotiate interactions based on appointment type is critical in light of recent studies suggesting that patient concerns (typically psychological in nature), were not adequately addressed by audiologists [8-10].

It has been suggested that good communication and supporting sharing decision-making are important factors in promoting trust in hearing healthcare service delivery [12]. However, there is limited emphasis on understanding the audiologist-patient communication behavior, although some recent studies exist [8-10]. Much effort is needed to educate students and practicing audiologists about appropriate means and form of communication, which could bring measurable benefit in terms of patient satisfaction and therefore compliance. Moreover, considering that the significant others of person with hearing loss are often involved in audiological con- 
sultation session and decision-making, it is also important to involve significant others in the communication during audiology consultation and rehabilitation session $[14,15]$.

While the present study presents some interesting observations, it has limitations. Firstly, the limited sample size could influence results, however, this limitation was deemed acceptable by the authors considering the pilot nature of the study. Secondly, using interactions of a single audiologist may reduce the generalizability of the study results. Future studies should include not only a greater number, but also a wide variety of clinical employment settings. Thirdly, since studying only initial appointments may not fully address the spectrum of possible patient centeredness levels that may exist during the continuum of care for this chronic condition, future research should include broader studies that include follow up care and measures patient adherence to treatment.

Additionally, further research should explore a greater variety of communication aspects to get a fuller view of the patient-audiologist relationship. Furthermore, in order to consider the patient perspective, studies should explore or include the patient's explicit goals for particular sessions and their perceptions of the studied appointments. Finally, qualitative analyses of interactions were conducted.

Results suggest that during initial audiological consultations related to hearing difficulties, the audiologist produced more utterances related to explicit description of symptoms, whereas when the sessions had greater complexity or were related to management and treatment, the audiologist produced more utterances related to the patient's interpretation of symptoms. Also, a more equitable distribution of words and utterances per turn are observed during initial consultation about hearing difficulties when compared to complex disorders and hearing aid consultation sessions, where the audiologist was dominant within the interaction. These findings provide unique insights to audiologist's communication behavior during audiology consultation sessions. Efforts are needed to educate and promote appropriate communication between audiologists' and patients, which could result in improved trust between them and ultimately resulting in increased patient satisfaction.

\section{Conflicts of interest}

The authors have no financial conflicts of interest.

\section{REFERENCES}

1) Epstein RM, Street RL Jr. The values and value of patient-centered care. Ann Fam Med 2011;9:100-3.

2) Street RL Jr, Gordon H, Haidet P. Physicians' communication and perceptions of patients: is it how they look, how they talk, or is it just the doctor? Soc Sci Med 2007;65:586-98.

3) Street RL Jr, Makoul G, Arora NK, Epstein RM. How does communication heal? Pathways linking clinician-patient communication to health outcomes. Patient Educ Couns 2009;74:295-301.

4) Kochkin S. MarkeTrak VIII: the efficacy of hearing aids in achieving compensation equity in the workplace. Hear J 2010;63:19-26.

5) Brown JB, Stewart M, Ryan BL. Assessing communication between patients and physicians: the measure of patient-centred communication (MPCC). London: Centre for Studies in Family Medicine;2001.

6) Lown BA, Clark WD, Hanson JL. Mutual influence in shared decision making: a collaborative study of patients and physicians. Health Expect 2009;12:160-74.

7) Street RL Jr. Information-giving in medical consultations: the influence of patients' communicative styles and personal characteristics. Soc Sci Med 1991;32:541-8.

8) Ekberg K, Grenness C, Hickson L. Addressing patients' psychosocial concerns regarding hearing aids within audiology appointments for older adults. Am J Audiol 2014;23:337-50.

9) Grenness C, Hickson L, Laplante-Lévesque A, Meyer C, Davidson B. Communication patterns in audiologic rehabilitation history-taking: audiologists, patients, and their companions. Ear Hear 2015;36: 191-204.

10) Grenness C, Hickson L, Laplante-Lévesque A, Meyer C, Davidson B. The nature of communication throughout diagnosis and management planning in initial audiologic rehabilitation consultations. J Am Acad Audiol 2015;26:36-50.

11) The Hearing Review. US hearing aid sales flat in first quarter of 2014 [cited 2016 Aug 8]. Available from: http://www.hearingreview. com/2014/04/us-hearing-aid-sales-flat-first-quarter-2014/.

12) MacWhinney B. The CHILDES project: tools for analyzing talk. 3rd ed. Mahwah, NJ: Lawrence Erlbaum Associates;2000.

13) Epstein RM, Franks P, Fiscella K, Shields CG, Meldrum SC, Kravitz $\mathrm{RL}$, et al. Measuring patient-centered communication in patientphysician consultations: theoretical and practical issues. Soc Sci Med 2005;61:1516-28.

14) Ekberg K, Meyer C, Scarinci N, Grenness C, Hickson L. Disagreements between clients and family members regarding clients' hearing and rehabilitation within audiology appointments for older adults. J Interact Res Commun Disord 2014;5:217-44.

15) Ekberg K, Meyer C, Scarinci N, Grenness C, Hickson L. Family member involvement in audiology appointments with older people with hearing impairment. Int J Audiol 2015;54:70-6. 